\title{
Evidencia de solapamiento de micro-hábitat entre juveniles del salmón introducido Oncorhynchus tshawytscha y el pez nativo Trichomycterus areolatus en el río Allipén, Chile
}

Evidence of microhabitat overlap between juvenile of introduced salmon Oncorhynchus tshawytscha and the native fish Trichomycterus areolatus in the Allipén River, Chile

\author{
Pamela V. Vargas ${ }^{1}$, Iván Arismendi ${ }^{2,3}$, Gladys Lara ${ }^{1}$, Javier Millar ${ }^{1}$ y Santiago Peredo ${ }^{1}$ \\ ${ }^{1}$ Escuela de Ciencias Ambientales, Facultad de Recursos Naturales, Universidad Católica de Temuco, Casilla D-15, Temuco, Chile. \\ pvargasaraya@gmail.com \\ ${ }^{2}$ Escuela de Graduados, Facultad de Ciencias Forestales, Universidad Austral de Chile, Casilla 567, Valdivia, Chile \\ ${ }^{3}$ USGS Forest and Rangeland Ecosystem Science Center, Aquatic Ecology Laboratory, 3200 SW Jefferson Way, Corvallis, Oregon \\ 97331, Estados Unidos
}

\begin{abstract}
Introduced juvenile Chinook salmon (Oncorhynchus tshawytscha) have been found co-occurring with native fishes in the Allipén River, Chile. Due to this co-occurence, the microhabitat use, microhabitat preferences, and overlap between juvenile Chinook salmon and the native catfish, Trichomycterus areolatus, were examined during the summer and fall of 2007-2008. Microhabitat preferences and overlap between juvenile Chinook salmon and the native catfish were determined using the Instream Flow Incremental Methodology and the Pianka's index. Juvenile Chinook salmon and the native catfish microhabitat preferences varied seasonally showing a high degree of similarity and overlap between the species (higher than $80 \%$ ). The results suggest the risk of negative interactions and interactive segregation over habitat processes between juvenile Chinook salmon and native catfish. As a consequence, the Chinook salmon invasion may threaten the stability of native catfish populations at Allipén River.
\end{abstract}

Key words: Biological invasions, co-occurrence, negative interactions, interactive segregation, temperate ecosystems

Resumen.- Juveniles del salmón Chinook (Oncorhynchus tshawytscha) introducido en el sur de Chile han sido observados co-ocurriendo con peces nativos en el río Allipén, Chile. Con objeto de identificar las potenciales implicancias ecológicas de esta invasión sobre la fauna íctica nativa, durante el verano y otoño de los años 2007-2008, se determinaron las preferencias y grado de solapamiento de microhábitat entre el salmón Chinook y el pez nativo bagre, Trichomycterus areolatus. Las preferencias y grado de solapamiento de microhábitat entre el salmón Chinook y el bagre se determinaron utilizando el método incremental para la asignación de caudales mínimos y el índice Pianka. En ambas especies, las preferencias de microhábitat variaron estacionalmente mostrando además un alto grado de similitud y solapamiento (mayor al 80\%). Los resultados obtenidos sugieren una potencial interacción negativa entre el salmón Chinook y el bagre a través de una segregación interactiva por hábitat. De este modo, el actual proceso de invasión del salmón Chinook en el sur de Chile representaría una amenaza para la estabilidad de las poblaciones de bagre en el río Allipén.

Palabras clave: Invasiones biológicas, co-ocurrencia, interacciones negativas, segregación interactiva, ecosistemas templados

\section{INTRODUCCIÓN}

El salmón Chinook, Oncorhynchus tshawytscha (Walbaum, 1792), tiene una distribución natural restringida al Hemisferio Norte. Es el salmónido de mayor tamaño en su tipo, con una longitud máxima sobre los $150 \mathrm{~cm}$ y un peso de hasta $45 \mathrm{~kg}$ (Healey 1991). Sin embargo, en los últimos años, se han establecido poblaciones autosostenidas de esta especie en cuencas hidrográficas de Sudamérica, específicamente en Chile y Argentina entre los $39^{\circ}$ y $53^{\circ}$ S (Soto et al. 2006, Becker et al. 2007, Soto et al. 2007, Correa \& Gross 2008, Astorga et al. 2008, Di Prinzio \& Pascual 2008). Una de las razones del éxito en el establecimiento de dichas poblaciones se debe a la alta plasticidad en el ciclo de vida del salmón Chinook (Ciancio et al. 2005, Soto et al. 2007, Arismendi 2009, Arismendi et al. 2009), característica que lo convierte en una especie invasora por excelencia. 
En el sur de Chile, a principios del otoño, adultos del salmón Chinook migran desde el mar hacia los ríos para reproducirse, lugar donde luego los juveniles crecen y se desarrollan hasta su retorno al mar a mediados del verano del año siguiente (Soto et al. 2007). Particularmente en el río Allipén, uno de los mayores tributarios de la cuenca del río Toltén $\left(38^{\circ} \mathrm{S}\right)$, se pueden encontrar juveniles del salmón Chinook co-ocurriendo con juveniles y adultos del pez nativo bagre (Trichomycterus areolatus Valenciennes, 1842).

El estudio de las interacciones ecológicas entre el salmón Chinook y los peces nativos es de gran importancia para la conservación de la fauna íctica autóctona del sur de Chile, dada la amenaza potencial que representa la introducción de salmonídeos en esta parte del mundo (Soto et al. 2006, Arismendi 2009, Arismendi et al. 2009). Especialmente relevante es el caso del bagre, catalogada como especie "vulnerable" a nivel nacional (Campos et al. 1998). En un estudio reciente, Penaluna et al. (2009) sugieren la existencia de segregación por microhábitat de las truchas, Oncorhynchus mykiss (Walbaum, 1792) y Salmo trutta (Linnaeus, 1758), hacia el bagre Trichomycterus areolatus. Sin embargo, aún no existen estudios que determinen las potenciales interacciones ecológicas, tales como depredación y competencia, existentes entre el salmón Chinook y dicha especie nativa (T. areolatus). Dado el corto período de tiempo en que los adultos del salmón Chinook se encuentran en los ríos del sur de Chile (Soto et al. 2007, Correa \& Gross 2008), es razonable pensar que las potenciales interacciones negativas con el bagre podrían derivarse de relaciones competitivas (alimento y/o hábitat) sólo con los juveniles del salmón Chinook.

De lo documentado sobre el nicho trófico, tanto para salmón Chinook en su estado juvenil (Randorf et al. 1990, Millar 2008) como para el bagre (Habit et al. 2005), se desprende que ambas especies son de hábitos omnívoros presentando además un amplio rango de ítems alimentarios en sus dietas. Por este motivo, es posible hipotetizar una baja probabilidad de interferencia negativa entre ambas especies por la vía de relaciones competitivas sólo por alimento. Sin embargo, un grado potencial de similitud en cuanto al uso del hábitat entre ambas especies aún no ha sido estudiado. Una mayor similitud o solapamiento de hábitat podría constituir un detonante de relaciones inter-específicas negativas, tales como la depredación, segregación por territorialidad y/o interferencia por explotación de recursos (Nilsson 1967).

En el presente estudio se determinaron las preferencias y grado de solapamiento de microhábitat entre el bagre y juveniles del salmón Chinook en un tramo del río Allipén, con el objetivo de determinar el potencial grado de interferencia entre ambas especies. En este estudio, se presenta información biológica relevante que puede servir como base para la identificación de potenciales riesgos para las especies nativas producto del actual proceso de invasión del salmón Chinook en el sur de Chile.

\section{Material y MÉtodos}

\section{Área de ESTUdio}

Los sitios de muestreo se localizaron en un tramo superior del río Allipén, sur de Chile (38 $51^{\circ} \mathrm{S}$ y $71^{\circ} 45^{`} \mathrm{~W}$ ) a una altura de $463 \mathrm{msnm}$. El río Allipén presenta un régimen hidrológico pluvio-nival ubicado en una zona bioclimática de tendencia mediterránea y con un clima templado lluvioso con precipitación media anual de $1.500 \mathrm{~mm}$ (Di Castri \& Hajek 1976). Para el presente estudio se seleccionó un tramo del río Allipén de $268 \mathrm{~m}$ de longitud y 11,5 m de ancho mojado. Los mesohábitats tipificados mostraron un patrón, aguas abajo, compuesto por pozón somero, pozón profundo, rápido, tabla, pozón somero, tabla y rápido. La relación rápidos/lentos fue de 0,25 con una distancia

Tabla 1. Número de individuos capturados por especie, rango de longitud total (LT) y época del año / Fish captured by species, total length (LT), and season

\begin{tabular}{clcc}
\hline Época del año & Especie & Captura & Rango de LT $(\mathrm{cm})$ \\
\hline \multirow{2}{*}{ Verano } & Salmón Chinook juvenil & 98 & $3,0-5,3$ \\
& Bagre juvenil & 54 & $2,9-4,9$ \\
& Bagre adulto & 36 & $5,2-13,0$ \\
\multirow{5}{*}{ Otoño } & & & \\
& Salmón Chinook juvenil & 133 & $3,6-5,7$ \\
& Bagre juvenil & 47 & $2,9-5,2$ \\
& Bagre adulto & 37 & $5,2-13,0$ \\
\hline
\end{tabular}


promedio entre rápidos de $173 \mathrm{~m}$ y una temperatura media del agua de $10^{\circ} \mathrm{C}$.

\section{USO, DISPONIBILIDAD Y PREFERENCIA DEL MICROHÁBITAT}

El uso, disponibilidad y preferencia de microhábitat para ambas especies, salmón Chinook y bagre, fue determinado mediante el empleo de la metodología incremental para la asignación de caudales mínimos, IFIM (Bovee et al. 1998). Se realizaron tres muestreos a principios del verano (diciembre) y tres a principios del otoño (marzo) durante los años 2007 y 2008. Los peces se capturaron a través de pesca eléctrica utilizando el método de dos pasadas consecutivas. El tramo seleccionado fue cerrado en ambos lados para evitar el escape de los peces. Los individuos capturados fueron clasificados por especie y estado de desarrollo (ver clasificación de Sommer et al. (2005) para juveniles del salmón Chinook, y Habit et al. (2005) para el bagre; Tabla 1). Una sub-muestra tomada al azar de los individuos capturados (29 en verano y 37 en otoño) fue utilizada para obtener información sobre el uso de microhábitat. En el lugar de detección de cada individuo capturado, se situó un marcador de posición donde se le midieron las siguientes variables de microhábitat: profundidad de la columna de agua $(\mathrm{cm})$, velocidad de corriente $\left(\mathrm{m} \mathrm{s}^{-1}\right)$, y tipo de refugio. Los tipos de refugio se clasificaron de la siguiente forma: (0) sin refugio, (1) algas, (2) vegetación acuática, (3) ramas y troncos sumergidos, (4) borde de elementos de sustrato, (5) cornisas sumergidas, (6) sombra, y (7) combinación de varios tipos de refugio. Posteriormente, para obtener la información correspondiente a la disponibilidad de microhábitat, se establecieron siete transectas perpendiculares al flujo del caudal en el tramo de río seleccionado donde fueron medidas aleatoriamente las mismas variables de microhábitat señaladas anteriormente (50 mediciones por transecta).

Para estimar la disponibilidad de cada variable de microhábitat (profundidad, velocidad de corriente, y tipo de refugio), se calculó la representatividad de cada transecta (i) como su aporte porcentual en longitud $\left(\mathrm{RT}_{i}\right.$ ) respecto del total de las transectas muestreadas. Luego, para cada transecta y de acuerdo al rango de valores de cada variable de microhábitat, se establecieron clases $(j)$, se estimó la frecuencia de cada clase $\left(\mathrm{F}_{j}\right)$ y se calculó la frecuencia ponderada multiplicando $\mathrm{F}_{j}$ por la representatividad de la transecta respectiva $\left(\mathrm{RT}_{i}\right)$. El índice de disponibilidad para cada clase “ $j$ ” $\left(\mathrm{I}_{\mathrm{dj}}\right)$ se estimó como el cuociente entre la suma de todos los valores de $\mathrm{F}_{j} \mathrm{x} \mathrm{RT}_{i}$ en todas las transectas y la suma de $\mathrm{F}_{j} \mathrm{xRT}_{i}$ para todas las clases en todas las transectas.

Para estimar el uso del microhábitat, se utilizó el índice de uso ( $\left.\mathrm{I}_{u j}\right)$ para cada clase " $j$ " definido como el cuociente entre el número de individuos asociados a una determinada clase “j” y el número total de individuos en el tramo muestreado. Finalmente, con los datos obtenidos del uso y disponibilidad de cada una de las variables de microhábitat, se procedió a calcular el índice de preferencia de microhábitat $\left(\mathrm{C}_{\mathrm{j}}\right)$ de acuerdo a la siguiente fórmula (Bovee et al. 1998):

$$
C_{j}=\frac{I_{u j}}{I_{d j}}
$$

\section{SOLAPAMIENTO EN PREFERENCIAS DE MICROHÁBITAT}

El solapamiento de microhábitat fue estimado mediante el índice de sobreposición de Pianka (1973) utilizando el módulo "niche overlap" del software Ecosim 7.0 (Gotelli \& Entsminger 2001). Para evaluar la significancia estadística de los resultados obtenidos se utilizó el test de permutaciones de Monte-Carlo. Este test crea un modelo de simulación nulo que representa las preferencias de microhábitat esperadas de ambas especies en ausencia de presión por competencia, el que luego se compara con las preferencias de microhábitat observadas. Para decidir si existen diferencias significativas se utilizaron 1000 permutaciones de las posibles preferencias esperadas y observadas, considerando un nivel de significancia del 5\% bajo la condición algorítmica Ra 3. Esta condicionante ha sido propuesta por Winemiller \& Pianka (1990) y es la más utilizada en la literatura. Las diferencias significativas que se reportan se deben interpretar como la existencia de una elevada similitud y solapamiento en las preferencias de microhábitat entre especies (e.g., Winemiller \& Pianka 1990).

\section{Resultados}

En ambas estaciones del año (verano y otoño) existió una mayor disponibilidad de microhábitats con profundidades entre 8 y $19 \mathrm{~cm}$, con bajas velocidades de corriente $(0,0$ $0,3 \mathrm{~m} \mathrm{~s}^{-1}$ ) y con los elementos de refugio correspondientes a los de borde de elementos del sustrato (Tabla 2). A su vez, entre los microhábitats con menor disponibilidad estuvieron aquellos más complejos (combinación de varios elementos de refugio), microhabitats con profundidades mayores a los $40 \mathrm{~cm}$ y los con velocidades de corriente mayor a $0,40 \mathrm{~m} \mathrm{~s}^{-1}$. 
Tabla 2. Disponibilidad de profundidad, velocidad y tipo de refugio. (0) sin refugio, (1) algas, (2) vegetación acuática, (3) ramas y troncos sumergidos, (4) borde de elementos de sustrato, (5) cornisas sumergidas, (6) sombra, y (7) combinación de varios elementos de refugio / Availability of depth, velocity, and in-stream cover type. (0) without in-stream cover, (1) algae, (2) aquatic vegetation, (3) submerged branches and trunks, (4) in-stream rocks, (5) submerged cornices, (6) shade, and (7) multiple cover types

\begin{tabular}{cccc}
\hline Disponibilidad (\%) & Profundidad (cm) & Velocidad $\left(\mathrm{m} \mathrm{s}^{-1}\right)$ & Tipo de refugio \\
\hline$<10$ & $40-69$ & $0,40-0,99$ & $1,2,3,5,6$ \\
$10-20$ & $30-39$ & $0,30-039$ & 7 \\
$21-40$ & - & $0,10-0,19$ & 0 \\
$41-60$ & $4-7$ & - & - \\
$61-80$ & $20-29$ & $0,20-0,29$ & - \\
$81-100$ & $08-19$ & $0,00-0,09$ & 4 \\
\hline
\end{tabular}

Los microhábitat más preferidos por el salmón Chinook y el bagre, medidos como profundidad de la columna de agua (Fig. 1), fueron los de profundidades entre 10 y $20 \mathrm{~cm}$ tanto en verano como en otoño. Por otro lado, en el caso de la velocidad de corriente en verano (Fig. 2), ambas especies prefirieron velocidades similares de no más de $0,30 \mathrm{~m} \mathrm{~s}^{-1} \mathrm{y}$ con una marcada preferencia por velocidades entre 0,1 y 0,2 $\mathrm{m} \mathrm{s}^{-1}$. Sin embargo, en otoño, tanto el salmón Chinook como el bagre tuvieron una mayor preferencia por microhábitats con velocidades de corriente mayores. Particularmente, los juveniles de ambas especies mostraron una preferencias bi-modal ( 0,2 y $0,6 \mathrm{~m} \mathrm{~s}^{-1}$; Fig. 2).

Las curvas de preferencias de microhábitat según el tipo de refugio indicaron que el salmón Chinook juvenil, tanto en verano como en otoño, prefirió los hábitats más complejos (combinación de varios elementos de refugio; Fig. 3). En el caso del bagre, en verano, los juveniles prefirieron casi exclusivamente la vegetación acuática, mientras que los adultos lo hicieron sobre el tipo de refugio denominado borde de diversos elementos de sustrato. La situación fue diferente en otoño, ya que tanto los juveniles como los adultos del bagre presentaron una marcada preferencia sólo por los hábitats más complejos.

El análisis de similitud de preferencias de microhábitats entre juveniles de salmón Chinook y el bagre (juveniles y adultos), en base al índice de Pianka, presentó valores superiores al $80 \%$ tanto en verano como en otoño, siendo incluso mayor al $90 \%$ en otoño. Ello se reflejó en un significativo solapamiento de microhábitat entre ambas especies, tanto en verano como en otoño $(P<0,001$; Tabla 3).

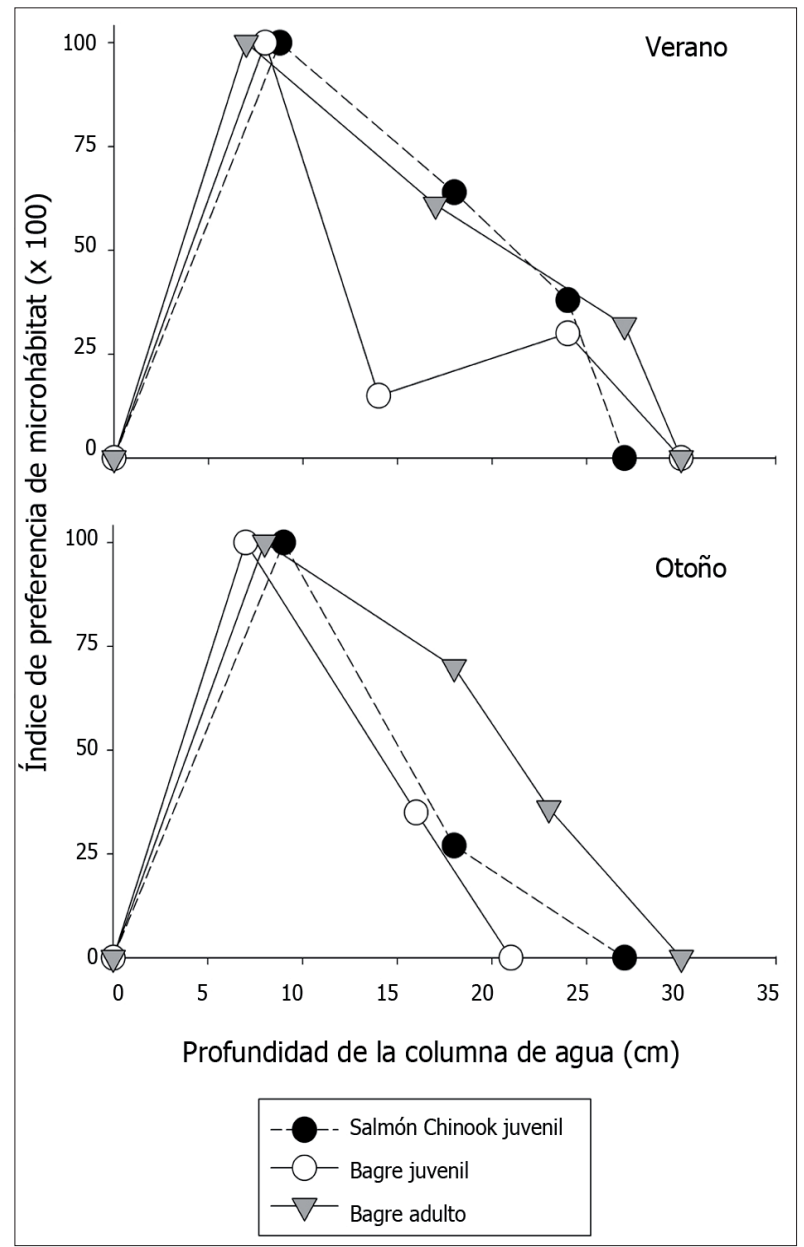

Figura 1. Preferencia de microhábitat, medido como profundidad de la columna de agua, en verano y otoño para juveniles del salmón Chinook, juveniles y adultos del bagre en el río Allipén / Water depth microhabitat preference in summer and fall for juvenile Chinook salmon and native catfish juvenile \& adult in Allipén River 


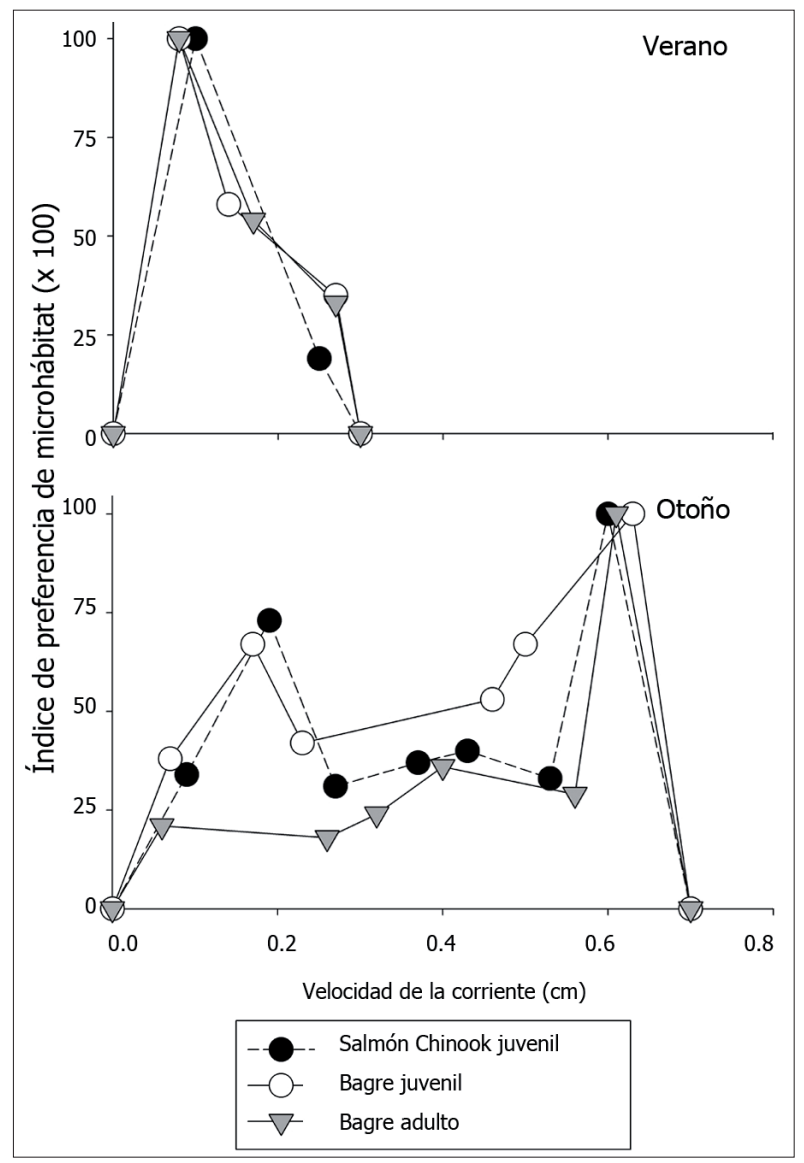

Figura 2. Preferencia de microhábitat, medido como velocidad de corriente, en verano y otoño para juveniles del salmón Chinook, juveniles y adultos del bagre en el río Allipén / Water velocity microhabitat preference in summer and fall for juvenile Chinook salmon and native catfish juvenile \& adult in Allipén River

\section{DisCUSIÓN}

Los resultados del presente estudio indican un elevado solapamiento en las preferencias de microhábitat entre juveniles del salmón Chinook y el pez nativo bagre, lo que sugiere una elevada superposición de nicho entre ambas especies en el verano y principios del otoño de cada año. Precisamente en esta época del año ocurre una menor precipitación (Di Castri \& Hajek 1976) y flujo de agua en los ríos de la zona y por ende, una disminución en la disponibilidad de microhábitats. Producto de ello, los hábitats más complejos resultaron ser los menos disponibles, aunque los más preferidos por parte de ambas especies. Este fenómeno sugiere un aumento de la posibilidad de competencia por territorialidad entre el salmón Chinook y el bagre, lo que podría reflejarse especialmente en verano donde se aprecia un aparente desplazamiento de los bagres desde microhábitats más complejos hacia otros menos preferidos. Dicho desplazamiento hacia hábitats menos óptimos ha sido reportado en otros ríos del sur de Chile por Penaluna et al. (2009) y descrito como un mecanismo de interacción negativa por parte de otros salmonídeos (Onchorhynchus mykiss y Salmo trutta) hacia el bagre T. areolatus. Análogamente, los resultados del presente estudio muestran una menor disponibilidad (rango) de velocidades de corriente intermedia en el verano, lo que también sugiere potenciales interacciones negativas entre ambas especies dada su preferencia similar en esta variable

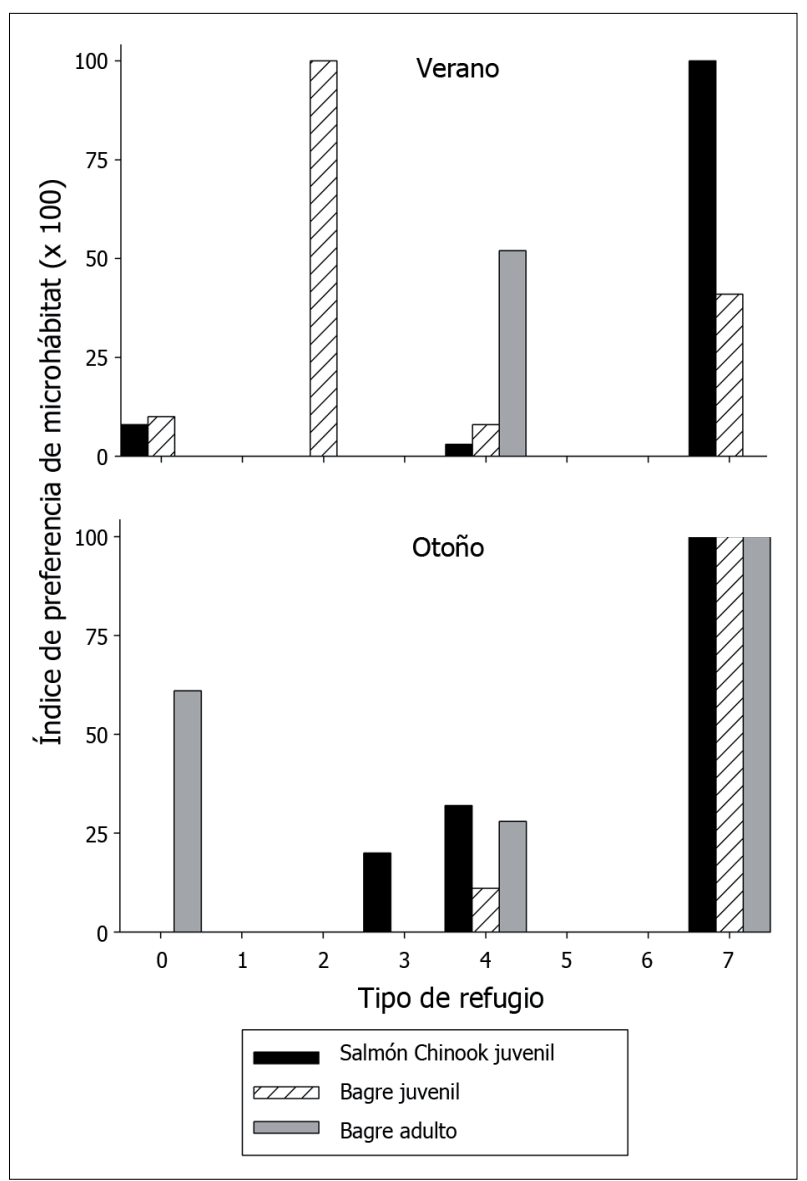

Figura 3. Preferencia de microhábitat, medido como tipo de refugio en verano y otoño para juveniles del salmón Chinook $y$ adultos del bagre en el río Allipén. Tipos de refugio: (0) sin refugio, (1) algas, (2) vegetación acuática, (3) ramas y troncos sumergidos, (4) borde de elementos de sustrato, (5) cornisas sumergidas, (6) sombra, y (7) combinación de varios elementos de refugio / Cover type microhabitat preference in: summer and fall for juvenile Chinook salmon and native catfish juvenile \& adult in Allipén River. In-stream cover types: (0) without in-stream cover, (1) algae, (2) aquatic vegetation, (3) submerged branches and trunks, (4) in-stream rocks, (5) submerged cornices, (6) shade, and (7) multiple cover types habitat 
Tabla 3. Grado de solapamiento de microhábitat y análisis de significancia entre datos observados (obs) y esperados (esp) (Pm: Probabilidad de que la media esperada sea mayor a la observada, Pv: Probabilidad de que la varianza esperada sea mayor que la observada) / Degree and significance of microhabitat overlap between observed (obs) and expected (esp) data (Pm: Probability that the expected mean is higher than the observed, Pv: Probability that the expected variance is higher than the observed)

\begin{tabular}{|c|c|c|c|c|c|c|c|c|c|}
\hline \multirow[b]{2}{*}{ Época del año } & \multirow[b]{2}{*}{ Especie } & \multicolumn{2}{|c|}{ Bagre } & \multicolumn{2}{|c|}{ Media } & \multicolumn{2}{|c|}{ Varianza } & \multicolumn{2}{|c|}{ Probabilidad } \\
\hline & & juvenil & adulto & obs & esp & obs & esp & $P m$ & $P v$ \\
\hline \multirow[t]{2}{*}{ Verano } & Chinook juvenil & 0,84 & 0,89 & 0,20 & 0,11 & 0,90 & 0,04 & $<0,0001$ & $<0,0001$ \\
\hline & Bagre juvenil & & 0,93 & & & & & & \\
\hline \multirow[t]{2}{*}{ Otoño } & Chinook juvenil & 0,94 & 0,96 & 0,18 & 0,08 & 0,09 & 0,03 & $<0,0001$ & $<0,0001$ \\
\hline & Bagre juvenil & & 0,97 & & & & & & \\
\hline
\end{tabular}

de microhábitat. Además, la estructura bi-modal de preferencias en velocidad de corriente encontrada para el bagre y el salmón Chinook está en acuerdo con lo reportado por Arratia (1983), donde se describe al bagre en su estado juvenil ocupando hábitats complejos a lo largo de la ribera de los ríos (menor velocidad de corriente), mientras que los adultos lo harían en sitios de corriente rápida. En su ambiente natural, el salmón Chinook ha sido descrito como una especie muy territorial y de comportamiento agresivo (Scott \& Grossman 1973, Healey 1991), lo que también se ha relacionado directamente con la velocidad de la corriente (Remiers 1968). Indirectamente y concordando con los resultados del presente estudio, otros autores indican que en alopatría, tanto el salmón Chinook como el bagre mantienen preferencias de hábitat similares (Healey 1980, Arratia 1983, Habit et al. 2005).

Una superposición en las preferencias de microhábitat entre el salmón Chinook y el bagre estaría en acuerdo con la hipótesis de potenciales interacciones negativas o procesos de segregación interactiva (ver Nilsson 1967) entre los peces nativos y los salmónidos introducidos el sur de Chile (Soto et al. 2006, Arismendi 2009, Arismendi et al. 2009, Penaluna et al. 2009). Dada la agresividad y voracidad del salmón Chinook (Scott \& Grossman 1973, Healey 1991) existe un potencial desplazamiento del bagre hacia hábitats de menor calidad, lo que limitaría la condición y salud de las poblaciones nativas en sitios donde co-ocurren ambas especies. Se debe tener en cuenta que las potenciales interacciones negativas entre el bagre y el salmón Chinook ocurrirían probablemente sólo durante la etapa juvenil del ciclo de vida de este último, con una duración de alrededor de un año (Soto et al. 2007, Correa $\&$ Gross 2008). A pesar de ello, en otros sistemas invadidos, los juveniles del salmón Chinook han sido descritos como posibles competidores y depredadores de especies nativas, debido a sus ventajas morfológicas y comportamiento agresivo en su etapa juvenil.

Considerando los antecedentes expuestos en el presente trabajo, sumado a los registros históricos de pescadores deportivos reportando la presencia de adultos del salmón Chinook desovando en el río Allipén desde hace más de 10 años (Salas com. pers.), se puede augerir que dicha especie mantendría poblaciones asilvestradas y auto-sostenidas en esta cuenca (ver también el caso del río Petrohué en Soto et al. 2007 y Astorga et al. 2008). Dicho establecimiento significa el ingreso al río Allipén de un nuevo invasor que aumentaría la amenaza de impactos negativos hacia las especies nativas por parte de la invasión previa, desde hace más de un siglo, de otros salmonídeos (principalmente truchas, Basulto 2003, Soto et al. 2006). No obstante el establecimiento del salmón Chinook en aguas continentales del sur de Chile es un proceso reciente (Soto et al. 2007, Correa \& Gross 2008), los resultados obtenidos por el presente estudio tienen el valor de ser los primeros que entregan información base para determinar los potenciales impactos de dicha invasión sobre la fauna íctica nativa del sur de Chile. Nuevos estudios son necesarios que involucren estimaciones a nivel poblacional, especialmente considerando ríos con y sin la presencia de salmónidos (ver discusión en Soto et al. 2006). Además, aproximaciones experimentales y modelos de simulación podrían dar luces sobre cuáles serían los mecanismos más específicos y sus efectos a nivel del paisaje, que tendría la potencial segregación de las especies nativas en presencia del salmón Chinook.

A pesar de ser resultados preliminares y no conclusivos, la elevada superposición de preferencias de microhábitat entre el salmón Chinook y el bagre debería alertar sobre los riesgos asociados a la introducción de especies en 
aguas continentales del sur de Chile. Una revisión de los criterios relativos a la selección y puesta en marcha de las medidas actuales de protección de las especies salmonídeas versus la fauna íctica nativa es urgente. Además, se requiere urgentemente establecer los criterios científicos para determinar cuáles son las estrategias de conservación más efectivas para la protección futura de las especies nativas en el sur de Chile.

\section{Agradecimientos}

Esperanza Parada a través del proyecto DGI 20065 financió parte de esta investigación. Patricio Salas, guía de pesca con mosca, nos acompañó en la búsqueda del salmón Chinook y bagres en el río Allipén. Brooke Penaluna proporcionó comentarios y sugerencias muy útiles en versiones preliminares del manuscrito. Dos revisores anónimos mejoraron sustancialmente la versión final del manuscrito. I. Arismedi fue financiado a través de la beca CONICYT para estudiantes de doctorado.

\section{LiTERATURA CITADA}

Arismendi I. 2009. The success of non-native salmon and trout in southern Chile: human, environmental and invader dimensions in a conceptual model of biological invasion processes. Tesis Doctoral, Facultad de Ciencias Forestales, Universidad Austral de Chile, Valdivia, 157 pp.

Arismendi I, D Soto, B Penaluna, C Jara, C Leal \& J León-Muñoz. 2009. Aquaculture, non-native salmonid invasions, and associated declines of native fishes in lakes of the northern Chilean Patagonia. Freshwater Biology 54: 1135-1147.

Arratia G. 1983. Preferencias de hábitat de peces siluriformes de aguas continentales de Chile (Fam. Diplomystidae y Trichomycteridae). Studies on Neotropical Fauna and Environment 18(4): 217-237.

Astorga MP, C Valenzuela, I Arismendi \& JL Iriarte. 2008. Naturalized Chinook salmon in the northern Chilean Patagonia: Do they originate from salmon farming? Revista de Biología Marina y Oceanografía 43(3): 669-674.

Basulto S. 2003. El largo viaje de los salmones: una crónica olvidada, propagación y cultivo de especies acuáticas en Chile, 289 pp. Editorial Maval, Santiago de Chile.

Becker L, M Pascual \& N Basso. 2007. Colonization of the southern Patagonia Ocean by exotic Chinook salmon. Conservation Biology 21(5): 1347-1352.

Bovee KD, BL Lamb, JM Bartholow, CB Stalnaker, J Taylor \& J Henriksen. 1998. Stream habitat analysis using the instream flow incremental methodology, 131 pp. Biological Resources Division Information and
Technology Report USGS/BRD-1998-0004, United States Geological Survey Fort Collins, Denver.

Campos H, G Dazarola, B Dyer, L Fuentes, J Gavilán, L Huaquín, G Martínez, R Meléndez, G Pequeño, F Ponce, V Ruiz, W Sielfeld, D Soto, R Vega \& I Vila. 1998. Categorías de conservación de peces nativos de aguas continentales de Chile. Boletín, Museo Nacional de Historia Natural 47: 101-222.

Ciancio JE, MA Pascual, J Lancelotti, C Rossi \& F Botto. 2005. Natural colonization and establishment of a Chinook salmon Oncorhynchus tshawytscha, population in the Santa Cruz River, an Atlantic basin of Patagonia. Environmental Biology of Fishes 74: 219-227.

Correa C \& MR Gross. 2008. Chinook salmon invades southern South America. Biological Invasions 10: 615-639.

Di Castri F \& ER Hajek. 1976. Bioclimatología de Chile, 129 pp. Ediciones Universidad Católica de Chile, Santiago.

Di Prinzio CY \& MA Pascual. 2008. The establishment of exotic Chinook salmon (Oncorhynchus tshawytscha) in Pacific rivers of Chubut, Patagonia, Argentina. Annales de Limnologie - International Journal of Limnology 1: 61-68.

Gotelli NJ \& GL Entsminger. 2001. EcoSim: Null models software for ecology. Version 7.0. Acquired Intelligence Inc. \& Kesey-Bear. Jericho, VT 05465 [en línea] <http:// homepages.tog-ether.net/ gentsmin/ecosim.htm >

Habit E, P Victoriano \& H Campos. 2005. Ecología trófica y aspectos reproductivos de Trichomycterus areolatus (Pisces, Trichomycteridae) en ambientes lóticos artificiales. Revista de Biología Tropical 53: 195-210.

Healey MC. 1980. Utilization of the Nanaimo River Estuary by juvenile Chinook salmon (Oncorhynchus tshawytscha). Fishery Bulletin 77(3): 653-668.

Healey MC. 1991. Life history of Chinook salmon Oncorhynchus tshawytscha. En: Groot C \& L Margolis (eds). Pacific salmon life histories, pp. 113-393. University of British Columbia Press, Vancouver.

Millar J. 2008. Estudio del contenido gástrico del salmon Chinook(Oncorhynchus tshawytscha Walbaum, 1792), para determinar su dieta y aportar al conocimiento de su impacto sobre especies nativas de aguas continentales. Tesis de Licenciado en Recursos Naturales, Facultad de Recursos Naturales, Universidad Católica de Temuco, Temuco, 52 pp.

Nilsson NA. 1967. Interactive segregation between fish species. En: Gerking SD (ed). The biological basis of freshwater fish production, pp. 295-313. Wiley, New York.

Penaluna BE, I Arismendi \& D Soto. 2009. Evidence of interactive segregation between introduced trout and native fishes in northern Patagonia Rivers, Chile. Transactions of the American Fisheries Society 138: 839-845. 
Pianka ER. 1973. The structure of lizard communities. Annual Review of Ecology and Systematics 4: 53-74.

Randorf D, G Gray \& R Fairley. 1990. Feeding ecology of subyearling Chinook salmon in riverine and reservior habitats of the Columbia River. Transactions of the American Fisheries Society 119(1): 16-24.

Remiers P. 1968. Social behaviour among juvenile fall Chinook salmon. Journal of the Fisheries Research Board of Canada 25: 2005-2008.

Scott W \& E Grossman. 1973. Freshwater fishes of Canada. Bulletin of the Fisheries Research Board of Canada 184: 1-966.

Sommer R, W Harrell \& M Nobriga. 2005. Habitat use and stranding risk of juvenile Chinook salmon on a seasonal floodplain. North American Journal of Fisheries Management 25: 1493-1504.
Soto D, I Arismendi, J González, J Sanzana, F Jara, C Jara, E Guzmán \& A Lara. 2006. Sur de Chile, país de truchas y salmones: Patrones de invasión y amenazas para las especies nativas. Revista Chilena de Historia Natural 79: 97-117.

Soto D, I Arismendi, C Di Prinzio \& F Jara. 2007. Establishment of Chinook salmon (Oncorhynchus tshawytscha) in pacific basins of southern South America and its potential ecosystem implications. Revista Chilena de Historia Natural 80: 61-65.

Winemiller K \& E Pianka. 1990. Organization in natural assemblages of desert lizards and tropical fishes. Ecological Monographs 60: 27-55.

Recibido el 14 de agosto de 2009 y aceptado el 12 de mayo de 2010 